\title{
National Early Warning Score Parameters at the Emergency Department as In Hospital Cardiac Arrest Predictors
}

\author{
Samsul Maarif ${ }^{1}$, Teguh Wahju Sardjono ${ }^{2}$, Yulian Wiji Utami ${ }^{3}$ \\ ${ }^{1}$ Master of Nursing Program, Faculty of Medicine, Brawijaya University, Indonesia \& Aisyiyah \\ Islamic Hospital Malang, Indonesia \\ ${ }^{3}$ Medical Program, Faculty of Medicine, Brawijaya University, Indonesia \\ ${ }^{4}$ Nursing Program, Faculty of Medicine, Brawijaya University, Indonesia \\ Email: samsulmaarifmlg@yahoo.com
}

\begin{abstract}
In Hospital Cardiac Arrest (IHCA) is fairly common occurrence, although it can be prevented. Physiological status monitoring at Emergency Departement (ED) is crucial for early detection of potential IHCA incidence. National Early Warning Score (NEWS) is a scoring system to assess deterioration of patient's condition, but it is not yet known which parameters that have predictive value for IHCA incidence. Examine NEWS parameters of the patients while at the ED that have predictive value of IHCA incidence. This study was conducted retrospectively on inpatient medical records. The NEWS parameters examined were respiration rate score, oxygen saturation score, body temperature score, systolic blood pressure score, pulse rate score and level of consciousness score. Logistic regression analysis was used to test the predictive ability of NEWS parameters. Total score NEWS proved to be correlated with IHCA incidence $(p=0.000 ; r=0.434)$. Parameters that have predictive value are systolic blood pressure score $(p=0.001 ; O R=14.730)$, respiration rate score $(p=0.000 ; O R=14.483)$ and level of consciousness score $(p=0.000$; $\mathrm{OR}=6.920$ ). The NEWS parameter when the patients will be transferred from ED to the wards that have predictive value for IHCA incidence are systolic blood pressure score, respiration rate score and level of consciousness score.
\end{abstract}

Keywords: Cardiac Arrest, Early Warning Score, Predictor.

\section{A. INTRODUCTION}

Cardiac arrest is a condition of cessation electrical and mechanical activity of the heart that cause a person to fall, loss of consciousness and death (Graham, McCoy, \& Schultz, 2015). If the condition is experienced by patients undergoing hospitalization, it is called In Hospital Cardiac Arrest (IHCA). The incidence of IHCA is reported at 1.6 per 1000 patients in United Kingdom (UK) and Northern Ireland with a survival rate of $18.4 \%$ (Nolan et al., 2014). In the United States the incidence of IHCA reaches 290,000 cases every year or 9-10 cases per 1000 hospitalization with a survival rate of $24 \%$ (Andersen, Holmberg, Berg, Donnino, \& Asger Granfeldt, 2019).

Actually $62 \%$ of IHCA incidence can potentially be prevented. The occurrence of IHCA is caused largely by late detecting hemodynamic abnormalities so there is delay in conducting management (Merchant et al., 2011). Identification of changes in physiological status is very important in efforts to prevent the IHCA incidence. 
Alterations in physiological status can be identified through monitoring vital signs, laboratory examination results and electrocardiogram (ECG). Alterations in vital signs are the simplest and easiest variable and can be detected quickly.

The NEWS is a scoring system used to identify alterations in the physiological status of hospitalization patients. The scoring system was used in the UK (England, Scotland and Northern Ireland) since 2012. This instrument is used for adult patients using six parameters as a basis for the change in physiological status assessment including respiration rate, oxygen saturation, systolic blood pressure, pulse rate, temperature and level of consciousness (Royal College of Physicians, 2012). Scores are given for each parameter and are subsequently merged into the total score in NEWS. The score in each NEWS parameter reflects the magnitude of a change from the normal value of that parameter.

The NEWS parameters can fluctuate rapidly especially in emergency conditions. Emergency patients are persons who are in the threat of death or who require immediate medical action (Kementrian Kesehatan Republik Indonesia, 2016). Patients who come to ED are in a state of unstable physiological status. Risk of a decrease in physiological status can occur up to 38 hours after the patient is admitted to the hospital through the ED (Considine, Jones, Pilcher, \& Currey, 2016). The deterioration in physiological status could potentially lead to IHCA incidence.

Basically, IHCA can be identified since the patient is in ED. The most frequent physiological abnormalities of patients in the ED that associated with IHCA are the pulse rate and level of consciousness (Considine et al., 2016). In contrast to other studies, patients who experienced IHCA, diastolic blood pressure was a physiological parameter that was detected abnormally at hospital admission (Churpek et al., 2012). Alterations in the respiratory rate and pulse rate are also detected at 48 hours before the onset of IHCA. Abnormalities of the patient's physiological status while in the ED related to the IHCA event are still being debated and need further research. This study purposes to retrospectively identify the most dominant NEWS parameters when the patient will be transferred from ED to the wards that can be used as an IHCA predictor.

\section{B. METHOD}

This observational study was conducted retrospectively with a case-control design of the medical record data of patients undergoing hospitalization at Aisyiyah Islamic Hospital Malang. Data was obtained from the medical records of hospitalization patients during the period of October 1, 2019 to January 31, 2020. Sample selection was carried out non-probability sampling with a consecutive sampling technique for all hospitalization patients admitted through ED. The inclusion criteria determined were medical records of hospitalization patients over the age of 16 years. Exclusion criteria were medical records of patients with pregnancy and postpartum, patients who were declared Do Not Resuscitate (DNR), and patients who experienced IHCA in the ED or during cardiac catheterization. 
During study period, there were 89 cases of IHCA; 73 of these cases met the inclusion criteria and were then taken as a sample of case groups. The control group was taken based on inclusion and exclusion criteria and did not experience IHCA. Based on the adequacy of the sample calculation, taken 85 medical records documents so that the overall study involved 158 samples of medical record documents. The physiological status data taken are NEWS total score and component score of NEWS parameters which includes respiration rate score, oxygen saturation score, body temperature score, systolic blood pressure score, pulse rate score, and level of consciousness score. Spearman analysis was used to test the correlation of six NEWS parameters to the incidence of IHCA. Logistic regression analysis was used to test the ability of the six NEWS parameters as predictors of IHCA incidence.

\section{RESULT AND DISCUSSION}

The incidence of IHCA in men (48.2\%) was higher than in women (43.8\%), but the difference was not significant $(\mathrm{p}=0.583)$ (Figure 1). The incidence of IHCA in both gender groups increased with age $(p=0.047 ; r=0.158)$. The age group over 66 years experienced the most common IHCA (55.6\%), followed by $41-65$ years $(45.4 \%)$, and $18-40$ years $25.0 \%$ (Figure 2 ).

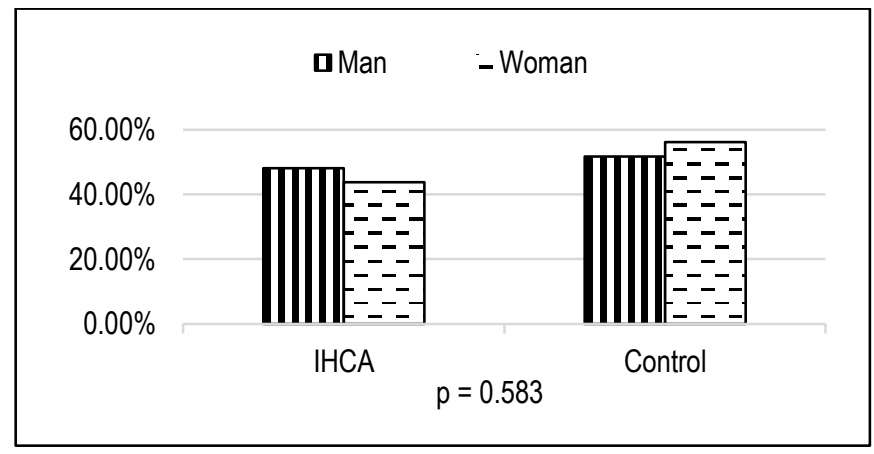

Figure 1 IHCA Incidence Distribution Graph Based on Gender

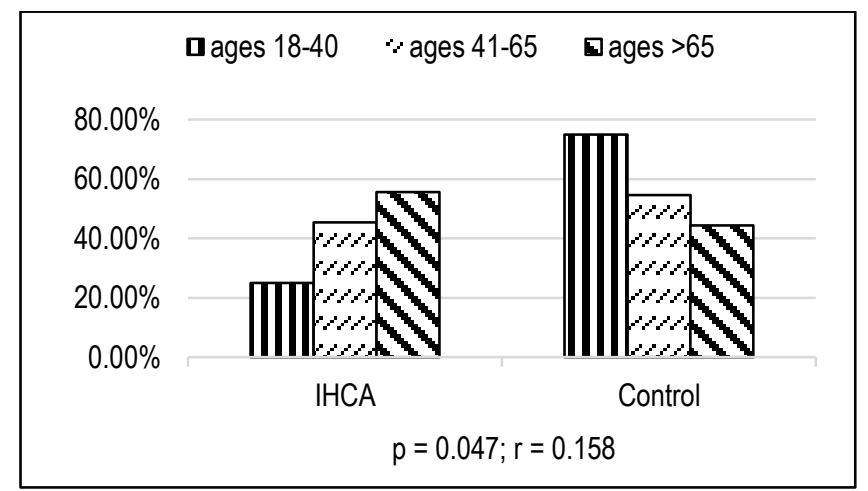

Figure 2 IHCA Incidence Distribution Graph Based on Ages

The total score NEWS when the patient will be transferred from ED to the wards represented a significant correlation to the IHCA incidence $(P=0.000 ; r=$ 
0.434). On the high score NEWS (score $\geq 7$ ) IHCA occurred up to $87.5 \%$ and $32.1 \%$ in the low score NEWS (score $\leq 4$ ) (Figure 3).

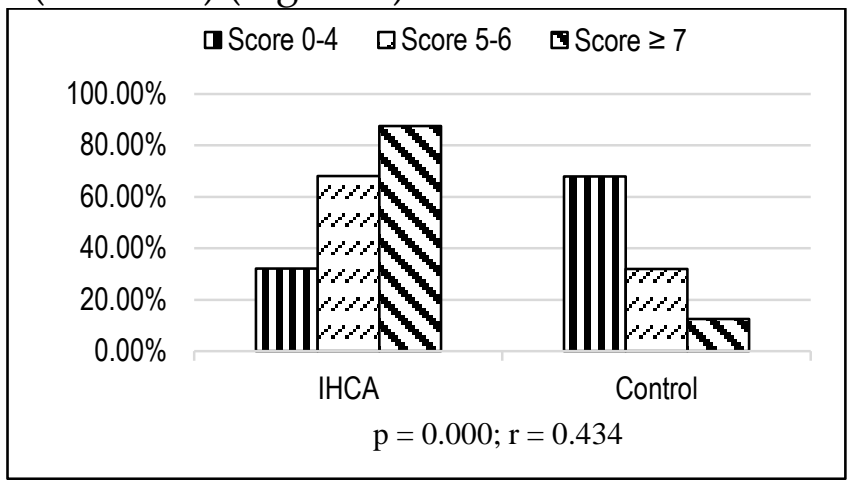

Figure 3 IHCA Incidence Distribution Graph Based on Total Score NEWS

A bivariate test was conducted to determine the correlation of six NEWS parameters when the patient will be transferred from the ED to the wards for IHCA incidence. The analysis represented a correlation of four NEWS parameters to the IHCA incidence. These parameters include the respiration rate score $(p=0.000 ; r=$ $0.434)$, oxygen saturation score $(\mathrm{p}=0.006 ; \mathrm{r}=0.220)$, systolic blood pressure score $(\mathrm{p}$ $=0.004 ; r=0.229)$, and the level of consciousness score $(p=0.000 ; r=0.307)$ (Table 1$)$.

Table 1 Bivariate Analysis Result of NEWS Parameters

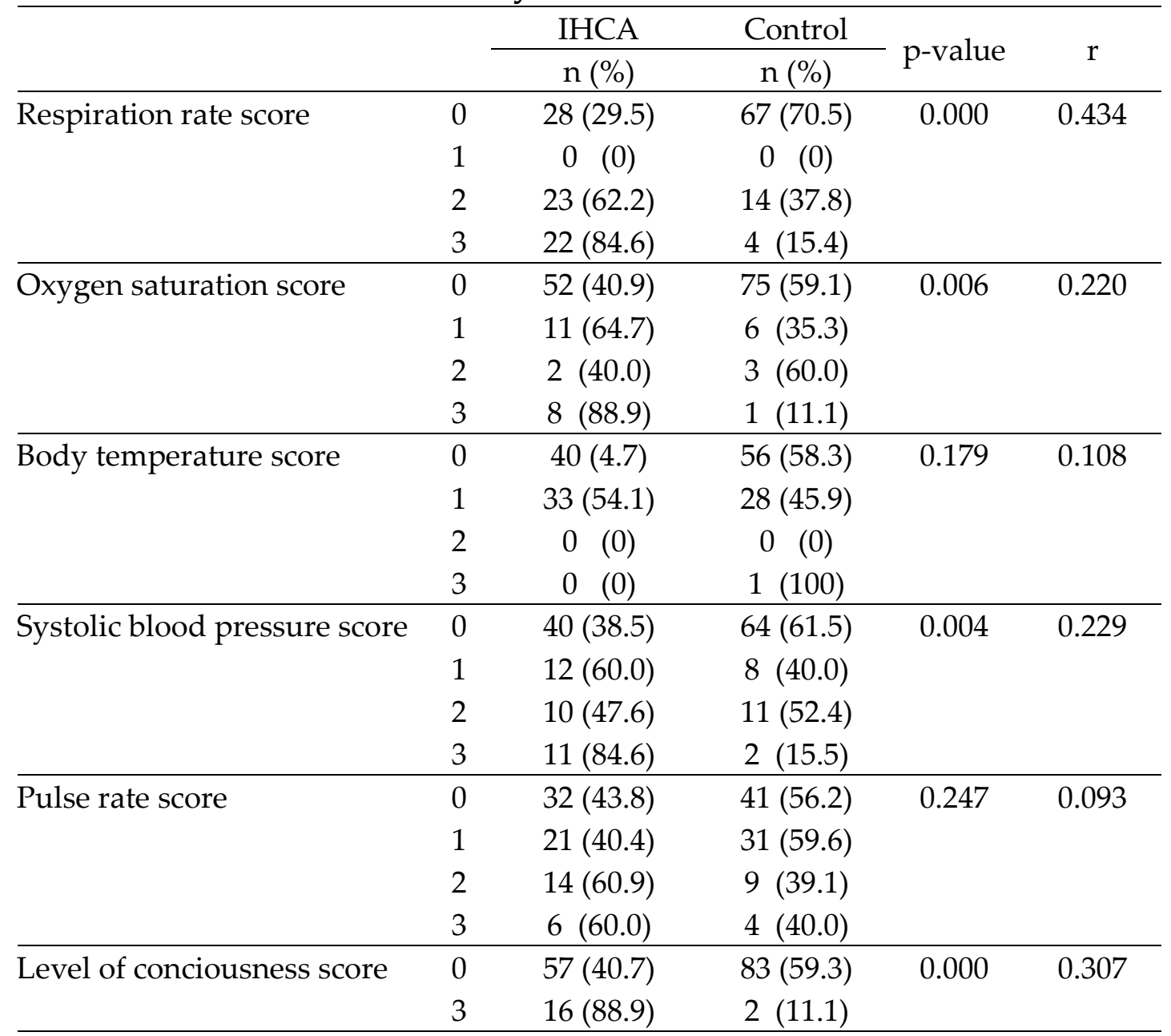


The bivariate test represented that all parameters had $\mathrm{p}$-value of $<0.25$, so that they were declared eligible for a multivariate test. Through the backward method of logistic regression test, body temperature score, pulse rate score and oxygen saturation score were excluded from the analysis because they did not have predictive ability. The remaining three parameters are respiration rate score, systolic blood pressure score and level of consciousness score. The parameters that have a predictive ability on the IHCA incidence are a score of 2 respiratory rates $(p=0.003$; $\mathrm{OR}=3.780)$, a score of 3 respiratory rates $(\mathrm{p}=0.000 ; \mathrm{OR}=14.483)$, a score of 3 systolic blood pressure $(\mathrm{p}=0.001 ; \mathrm{OR}=14.730)$ and a score of 3 levels of conciousness $(\mathrm{p}=$ $0.020 ; \mathrm{OR}=6.920$ ).

Table 2 Multivariate Analysis Result

\begin{tabular}{lccccc}
\hline & \multirow{2}{*}{ Coeff. } & p-value & \multirow{2}{*}{ OR } & \multicolumn{2}{c}{ CI 95\% } \\
\cline { 5 - 6 } & & & & Min. & Max. \\
\hline Score 2 respiration rate & 1.330 & 0.003 & 3.780 & 1.566 & 9.125 \\
Score 3 respiration rate & 2.673 & 0.000 & 14.483 & 4.279 & 49.017 \\
Score 1 systolic blood pressure & 1.050 & 0.063 & 2.857 & 0.946 & 8.634 \\
Score 2 systolic blood pressure & 0.462 & 0.417 & 1.588 & 0.520 & 4.851 \\
Score 3 systolic blood pressure & 2.690 & 0.001 & 14.730 & 2.821 & 76.908 \\
Score 3 level of consciousness & 1.934 & 0.020 & 6.920 & 1.349 & 35.512 \\
Constanta & -1.455 & 0.000 & 0.233 & & \\
\hline
\end{tabular}

Multivariate test results a constant value of -1.455 with the coefficients as presented in table 2. The equation formula from the constant values and the coefficient values of each variable is $y=-1.455+1.330$ (respiration rate 2) +2.673 (respiration rate 3$)+2.690$ (systolic blood pressure 3$)+1.934$ (level of consciousness) The quality of the equation was proven to have good calibration through the hosmer and lame show test $(\mathrm{p}=0.940)$ as well as good discrimination (AUC $=80.1 \%$ ). IHCA probability is determined by the formula $\mathrm{p}=1 /(1+\exp (-\mathrm{y}))$. The predicted value or HCA probability obtained based on the equation can be observed in the following table.

Table 3. Probability values of IHCA

\begin{tabular}{cccc}
\hline Respiration rate & Systolic blood pressure & Level of conciousness & Probability \\
\hline 0 & 0 & 0 & $18.923 \%$ \\
0 & 0 & 3 & $61.751 \%$ \\
3 & 0 & 0 & $77.171 \%$ \\
0 & 3 & 0 & $77.469 \%$ \\
3 & 0 & 3 & $95.899 \%$ \\
0 & 3 & 3 & $95.965 \%$ \\
3 & 3 & 0 & $98.031 \%$ \\
3 & 3 & 3 & $99.710 \%$ \\
\hline
\end{tabular}

This study involved 158 samples divided into case groups and control groups. The case group was 73 samples of medical records of patients who experienced IHCA. The incidence of IHCA was more common in men, but the difference was not 
statistically significant $(\mathrm{p}=0.583)$. In men, IHCA occurred at $48.2 \%$, while in women at $43.8 \%$. This is relevant to previous study which states that during the period 2003 - 2012, in the United States IHCA increased by $19.8 \%$ in men and $7.4 \%$ in women (Kim et al., 2016). Risk factors for cardiac arrest in men such as smoking, dietary habit and imbalance exercise patterns to cause cardiac arrest.

The incidence of IHCA increased with age $(p=0.047 ; \mathrm{r}=0.158)$. In the young adult group IHCA occurs by $25 \%$ and increases to $55.6 \%$ in elderly adult. Figure 2 shows that compared to young adulthood, the increase in the incidence of IHCA occurred up to two times greater in elderly adult. This is relevant to previous study which states that the highest incidence of IHCA is experienced by the age $\geq 71$ years. At $\geq 71$ years old IHCA is experienced by $50 \%$ of man and $60 \%$ of woman patients (Qvick et al., 2018). The presence of comorbidities suffered by the old age group is the reason for the high IHCA incidence in this group.

The total score NEWS of patients when they will be transferred from the ED to the wards showed a significant correlation with the incidence of IHCA ( $p=0.000$; $\mathrm{r}=0.434$ ). Patients with a total NEWS score of 5-6 (moderate score) had a $68 \%$ risk of IHCA. At a total score $\geq 7$ (high score) IHCA increased to $87.5 \%$ or three times higher than the low score. Previous studies have shown that patients with a total score of NEWS $>7$ during pre hospital, had a risk of death 48 hours after being admitted to the hospital, four times greater than the score $\leq 4$ (Abbott et al., 2018). Death has also increased as the abnormalities of vital signs before cardiac arrest (Andersen et al., 2016).

The Spearman test for each NEWS parameter showed a correlation between the patient's respiration rate score when they will be transferred from ED to the wards for the IHCA incidence ( $p=0.000 ; r=0.434)$. Logistic regression analysis also showed that respiration rate scores had a predictive ability for the incidence of IHCA. The predictors of IHCA were score of 2 respiration rate $(p=0.003 ; \mathrm{OR}=3.780$; $\mathrm{CI}=95 \%)$ and score of 3 respiration rate $(\mathrm{p}=0.000 ; \mathrm{OR}=14.483 ; \mathrm{CI}=95 \%)$. Previous study was mentioned that the maximum respiration rate was the most accurate predictor of IHCA incidence (Churpek et al., 2012). Respiratory rate trends were the best predictor of clinical outcomes (Kellett, Murray, Woodworth, \& Huang, 2015). Respiration rate fluctuation are physiological responses to restore blood oxygen levels within normal limits to meet tissue oxygen demand. In contrast to oxygen saturation scores, although the bivariate analysis showed a correlation to IHCA incidence $(\mathrm{p}=0.006 ; \mathrm{r}=0.220)$, this parameter was not proven to have predictive strength.

Other NEWS parameters that correlate to IHCA incidence are the systolic blood pressure score $(\mathrm{p}=0.004 ; \mathrm{r}=0.229)$ and the level of consciousness score $(\mathrm{p}=$ $0.000 ; \mathrm{r}=0.307)$. These parameters describe the condition of circulation and neurological function. This is relevant to previous study which states that systolic blood pressure is the most sensitive circulating function parameter to detect the risk of cardiac arrest (Fujiwara et al., 2016). Another abnormality of the patient's physiological status in ED that associated with the IHCA incidence is the level of 
conciousness (Considine et al., 2016). In the logistic regression test, the two parameters were proven to have a predictive ability for the IHCA incidence. The predictors of IHCA were a score of 3 systolic blood pressures $(p=0.001 ; \mathrm{OR}=14.730$; $C I=95 \%)$ and a score of 3 levels of consciousness ( $p=0.020 ; O R=6.920 ; C I=95 \%)$.

The NEWS parameter that did not indicate a correlation to IHCA incidence was the body temperature score $(\mathrm{p}=0.179 ; \mathrm{r}=0.108)$. An increase in body temperature score was not followed by an increase in the IHCA incidence. This is relevant to previous study which states that body temperature is a weak predictor of the IHCA incidence (Churpek et al., 2012). Body temperature did not change at 24 hours, 16 hours, and 8 hours before the IHCA incidence (Kim et al., 2016). This is possible because body temperature is a vital sign that does not describe the condition of respiratory function, circulatory function or neurological function. Body temperature indicates the condition of thermoregulation, thermo effector and the body's metabolic regulation functions. Abnormalities that occur before IHCA incidence are related to respiratory function, circulatory function, and neurological function (Fujiwara et al., 2016).

Another parameter that has no correlation to the IHCA incidence is the pulse rate score $(\mathrm{p}=0.247 ; \mathrm{r}=0.093)$. These results are different from previous studies which mention there are differences in pulse rate 48 hours before the IHCA incidence (Churpek et al., 2012). Abnormalities in the patient's physiological status in the ED related to the IHCA incidence are pulse rate (Considine et al., 2016). This difference may be caused by fluctuations in pulse rate that can occur immediately when the patients arrives at ED, before being transferred, until they arrive at the wards. Pulse rate abnormalities may occur when the patients arrive in the wards because the physiological abnormalities increases $12.2 \%$ compared to before they were transferred from ED (Considine et al., 2016).

\section{CONCLUSION}

The NEWS parameter scores that have a predictive ability on the IHCA incidence are systolic blood pressure score, respiration rate score and level of consciousness score. Patients with a score of 3 systolic pressure, a score of 3 respiration rate and a score of 3 at the level of consciousness when they will be transferred from the ED to the wards had a $99.7 \%$ chance of IHCA incidence. Assessment of NEWS parameters must be done carefully, especially on the three parameters score that have a predictive ability for the IHCA incidence.

\section{REFERENCES}

1. Abbott, T. E. F., Cron, N., Vaid, N., Ip, D., Torrance, H. D. T., \& Emmanuel, J. (2018). Pre-hospital National Early Warning Score (NEWS) is associated with inhospital mortality and critical care unit admission: A cohort study. Annals of Medicine and Surgery, 27, 17-21. https://doi.org/10.1016/j.amsu.2018.01.006

2. Andersen, L. W., Holmberg, M. J., Berg, K. M., Donnino, M. W., \& Asger Granfeldt. (2019). In-Hospital Cardiac Arrest: A Review. NCBI. 
https://doi.org/10.1001/jama.2019.1696

3. Andersen, L. W., Young, W., Chase, M., Berg, K. M., Mortensen, S. J., Moskowitz, A., ... Cocchi, M. N. (2016). The prevalence and significance of abnormal vital signs prior to in-hospital cardiac arrest. Resuscitation, 98, 112-117. https://doi.org/10.1016/j.resuscitation.2015.08.016

4. Churpek, M. M., Yuen, T. C., Huber, M. T., Park, S. Y., Hall, J. B., \& Edelson, D. P. (2012). Predicting cardiac arrest on the wards: a nested case-control study. Chest, 141(5), 1170-1176. https://doi.org/10.1378/chest.11-1301

5. Considine, J., Jones, D., Pilcher, D., \& Currey, J. (2016). Patient physiological status at the emergency department-ward interface and emergency calls for clinical deterioration during early hospital admission. Journal of Advanced Nursing, 72(6), 1287-1300. https://doi.org/10.1111/jan.12922

6. Fujiwara, S., Koike, T., Moriyasu, M., Nakagawa, M., Atagi, K., Lefor, A. K., \& Fujitani, S. (2016). A retrospective study of in-hospital cardiac arrest. Acute Medicine E Surgery, 3(4), 320-325. https://doi.org/10.1002/ams2.193

7. Graham, R., McCoy, M., \& Schultz, A. (2015). Strategies to improve cardiac arrest survival: a time to act. Institute of Medicine of the Nationale Academies, 3(2), 61-64. https://doi.org/10.1002/ams2.192

8. Kellett, J., Murray, A., Woodworth, S., \& Huang, W. (2015). Trends in weighted vital signs and the clinical course of 44,531 acutely ill medical patients while in hospital. Acute Medicine, 14(1), 3-9.

9. Kementrian Kesehatan Republik Indonesia. (2016). Peraturan Menteri Kesehatan Republik Indonesia Nomor 19 tahun 2016 tentang Sistem Penanggulangan Gawat Darurat Terpadu, 1-18. Retrieved from http://hukor.kemkes.go.id/uploads/produk_hukum/PMK_No._19_ttg_Sistem_Pe nanggulangan_Gawat_Darurat_Terpadu_.pdf

10. Kim, L. K., Looser, P., Swaminathan, R. V, Horowitz, J., Friedman, O., Shin, J. H., ... Feldman, D. N. (2016). Sex-Based Disparities in Incidence, Treatment, and Outcomes of Cardiac Arrest in the United States, 2003-2012. Journal of the American Heart Association, 5(6). https://doi.org/10.1161/JAHA.116.003704

11. Merchant, R. M., Yang, L., Becker, L. B., Berg, R. A., Nadkarni, V., Nichol, G., ... Investigators, A. H. A. G. W. T. G.-R. (2011). Incidence of treated cardiac arrest in hospitalized patients in the United States. Critical Care Medicine, 39(11), 24012406. https://doi.org/10.1097/CCM.0b013e3182257459

12. Nolan, J. P., Soar, J., Smith, G. B., Gwinnutt, C., Parrott, F., Power, S., ... Rowan, K. (2014). Incidence and outcome of in-hospital cardiac arrest in the United Kingdom National Cardiac Arrest Audit. Resuscitation, 85(8), 987-992. https://doi.org/10.1016/j.resuscitation.2014.04.002

13. Qvick, A., Radif, M., Brever, C., Myrvik, J. O., Schenk Gustafsson, K., \& Djärv, T. (2018). Survival of in-hospital cardiac arrest in men and women in a large Swedish cohort. Scandinavian Journal of Trauma, Resuscitation and Emergency Medicine, 26(1), 108. https://doi.org/10.1186/s13049-018-0576-0 
14. Royal College of Physicians. (2012). National Early Warning Score ( NEWS ), Standardising the assessment of acute-illness severity in the NHS. Retrieved from https://www.ombudsman.org.uk/sites/default/files/National Early Warning Score (NEWS) - Standardising the assessment of acute-illness severity in the NHS_0.pdf 